\title{
Estimation de la concentration en chlorophylles de feuilles par mesure de leur réflectance ou par analyse numérique de photographies prises au laboratoire
}

\author{
B Andrieu 1, S Kiriakos 1, KW Jaggard 2 \\ 1 INRA, Bioclimatologie 78850 Thiverval-Grignon, France; \\ 2 AFRC-IACR Broom's Barn IP28 6NP Bury St Edmunds, UK
}

(Reçu le 27 janvier 1992; accepté le 25 février 1992)

\begin{abstract}
Résumé - Des simulations effectuées avec un modèle de réflectance de feuille permettent de proposer une méthode simple d'estimation de la concentration en chlorophylles, fondée sur la mesure de la réflectance dans le rouge ou le vert. Les résultats obtenus sont évalués sur des feuilles de betteraves à sucre, en utilisant 2 capteurs adaptés à la mesure de la réflectance sur des échantillons de dimensions importantes:

- en mesurant directement la réflectance foliaire, avec un densitomètre photographique qui permet une mesure rapide mais ponctuelle $\left(5 \mathrm{~mm}^{2}\right)$;

- en estimant la réflectance par numérisation de photographies de feuilles prises au laboratoire, qui fournissent une image de la distribution spatiale des pigments.

Les résultats expérimentaux confirment l'existence d'une relation simple entre la réflectance et la concentration en chlorophylles; toutefois les paramètres ajustés expérimentalement pour cette relation diffèrent sensiblement de ceux calculés par le modèle. Cette différence semble provenir principalement de l'utilisation de capteurs à bande spectrale large. L'utilisation des capteurs proposés nécessite donc de disposer d'un échantillon d'apprentissage pour l'ajustement de 2 paramètres.
\end{abstract}

chlorophylle / feuille / réflectance / densité optique / photographie

\begin{abstract}
Summary - Estimation of chlorophyll content of leaves using reflectance measurements or digitalized photographs of leaves taken in the laboratory. A simple method based on model simulations of leaf reflectance was proposed for the estimation of leaf chlorophyll content from reflectance measurements in the red or green parts of the spectrum. The results were evaluated with sugar beet leaves using 2 methods to acquire the data. The methods were chosen for their ability to provide measurements on large samples: first, direct measurements of leaf reflectance were made using a photographic densitometer capable of taking quick measurements on small areas (5 $\left.\mathrm{mm}^{2}\right)$. Second, indirect estimates of leaf reflectance were made from digital analysis of color photographs of leaves taken in laboratory; this gave an image of the spatial distribution of pigments. Experimental results confirmed that a simple relation exists between reflectance and chlorophyll content. However, the experimental parameters of the relation significantly differed from those computed from the model. It seems that the difference is mainly due to the use of broad band sensors. Thus for operational use, it is necessary to calibrate 2 parameters on a sample of leaves of known chlorophyll content.
\end{abstract}

chlorophyll / leaf / reflectance / optical density / photograph 


\section{INTRODUCTION}

Les chloroses foliaires sont un symptôme fréquent de maladies, de carences, ou de sénescence. La caractérisation de ces symptômes se fait souvent sur la base de notations qualitatives, car le dosage des chlorophylles est une opération fastidieuse. De plus, les couverts chlorosés présentent généralement une forte hétérogénéité de pigmentation, qui pose le problème de la représentativité des mesures. Le besoin existe donc d'une méthode d'estimation des chloroses permettant d'analyser un grand nombre d'échantillons et de décrire la distribution spatiale de la concentration en chlorophylles (Eastwood et Steven, 1991). Dans cette perspective, on évalue ici une méthode fondée sur les relations entre réflectance et concentration en pigments.

La réflectance et la transmittance foliaire dépendent de l'absorption du rayonnement par les constituants chimiques et de sa diffusion par les tissus. Dans un précédent travail (Andrieu et Baret, 1988), nous avions utilisé un formalisme semi-empirique dérivé de la théorie de Kubelka-Munk pour étudier les relations entre propriétés optiques des feuilles et concentrations en pigments; nous évaluons ici une méthode simple d'estimation de la concentration en chlorophylles, fondée sur les résultats de simulations avec le modèle PROSPECT (Jacquemoud et Baret, 1991). Le modèle utilisé est une généralisation du «plate model» (Allen et al, 1969); il calcule les propriétés optiques des feuilles pour les longueurs d'onde comprises entre 400 et $2500 \mathrm{~nm}$, en fonction des concentrations surfaciques en pigments (caroténoïdes et chlorophylles) et en eau, et d'un paramètre traduisant la diffusion du rayonnement par les tissus.

Jacquemoud et Baret (1991) ont montré que leur modèle pouvait être inversé à partir des spectres de réflectance et de transmittance foliaire. Nous nous sommes intéressés à l'estimation de la concentration foliaire en chlorophylles à partir d'une seule mesure de réflectance dans le rouge ou le vert, et à la possibilité d'obtenir une image de la distribution des pigments dans la plante, en estimant indirectement la distribution des réflectances foliaires, par analyse numérique de photographies de feuilles prises en laboratoire.

\section{MATÉRIEL ET MÉTHODE}

\section{Simulations sur modèle}

La forme des relations entre réflectance et concentration en pigments, et la sensibilité de ces relations à la structure de la feuille ont été étudiées par simulations sur le modèle PROSPECT. Dans le cas des photographies de feuilles, on a étudié également l'influence de la réflectance du support sur ces relations. En effet, pour les feuilles à faible concentration en chlorophylles, le rayonnement transmis par la feuille et réfléchi par le support n'est pas négligeable devant le rayonnement directement réfléchi par la feuille.

\section{Protocole expérimental}

Les mesures ont été réalisées le 5 juillet 1989 et le 20 juillet 1990 sur des feuilles de betterave sucrière (Beta vulgaris) provenant de parcelles expérimentales. En 1989 , les chloroses résultent d'une infestation artificielle par le virus de la jaunisse (BYV), en 1990 elles sont obtenues en traitant les parcelles avec un désherbant ( 3 traitements, $\mathrm{HO}$ : témoin, $\mathrm{H} 1: 1,6 \mathrm{~g} / \mathrm{ha}$ de Metsulfuron méthyle, $\mathrm{H} 2: 2,4 \mathrm{~g} / \mathrm{ha}$ de Metsulfuron méthyle).

\section{Expérimentation de 1989}

On utilise un échantillon de 18 morceaux de feuilles, choisis pour être chacun de couleur relativement homogène, et tels que l'ensemble couvre la gamme de colorations existant sur les parcelles (vert à très jaune). Les feuilles sont prélevées sur des plantes différentes, et en différents endroits des bouquets foliaires. On exclut toutefois les feuilles nécrosées. La réflectance de chaque morceau de feuille est mesurée en laboratoire avec un densitomètre MAC-BETH, appareil habituellement utilisé pour mesurer la réflectance et la transmittance de documents photographiques. Ce densitomètre réalise des mesures dans 3 bandes spectrales (rouge, vert, bleu) correspondant à la norme photographique Status A (fig 1). L'appareil permet une mise en place rapide de l'échantillon et une mesure instantanée de la réflectance; toutefois chaque mesure est ponctuelle $\left(5 \mathrm{~mm}^{2}\right)$. On retient la moyenne de 5 répétitions par élément de feuille.

Pour chaque morceau de feuille, les concentrations en chlorophylles $a$ et $b\left(C_{a}\right.$ et $\left.C_{b}\right)$ et en caroténoïdes totaux $\left(X_{c}\right.$ : carotène + xantophylle) sont mesurées selon la méthode proposée par Lichtenthaler et Wellburn (1983), après extraction des pigments dans l'acétone. La valeur retenue est la moyenne de 6 estimations par morceau de feuille, réalisée chacune sur un disque de $1 \mathrm{~cm}^{2}$ découpé avec une pince calibrée. 


\section{Expérimentation de 1990}

Pour l'expérimentation de 1990 , la réflectance foliaire est mesurée indirectement, à partir de photographies de feuilles prises en laboratoire. Les clichés sont numérisés en trichromie (rouge, vert, bleu) avec un densitomètre scanneur. On présentera plus loin le protocole d'étalonnage permettant de calculer la réflectance foliaire à partir des mesures délivrées par le densitomètre scanneur. Chaque photographie représente une plante, dont toutes les feuilles sont détachées et disposées sur un support de dimensions utiles $0,8 m \times 1,2$ $\mathrm{m}$. Quatre lampes halogènes de $500 \mathrm{~W}$, situées à $2 \mathrm{~m}$ au-dessus du support sont placées de façon à assurer un éclairage aussi homogène que possible. On utilise une émulsion couleur inversible pour lumière artificielle (Fuji RTP 704). L'appareil photographique est placé à une hauteur de $1,80 \mathrm{~m}$ avec un objectif de focale 50 $\mathrm{mm}$. Soixante photographies représentant environ 30 $\mathrm{m}^{2}$ de tissus foliaires ont été analysées, à raison d'une mesure tous les $100 \mu \mathrm{m}$, correspondant à un échantillonnage tous les $4 \mathrm{~mm}$ sur les feuilles.

Trois des photographies représentent un échantillon de 15 morceaux de feuilles dont la concentration en chlorophylles est mesurée directement selon le même protocole que celui décrit pour 1989. Ces mesures seront utilisées pour évaluer les résultats.

\section{Étalonnage des mesures photographiques (expérimentation de 1990)}

La méthode repose sur l'estimation de la réflectance foliaire dans le rouge ou le vert. La réflectance dans une bande spectrale peut être estimée en utilisant un film noir et blanc et un filtre pour limiter la bande de sensibilité de l'émulsion. Nous avons toutefois utilisé un film couleur, qui facilite l'identification automatique des feuilles par rapport au support sur lequel elles sont disposées.

Un film photographique couleur peut être considéré schématiquement comme constitué de 3 couches, sensibles respectivement au bleu, au vert, et au rouge

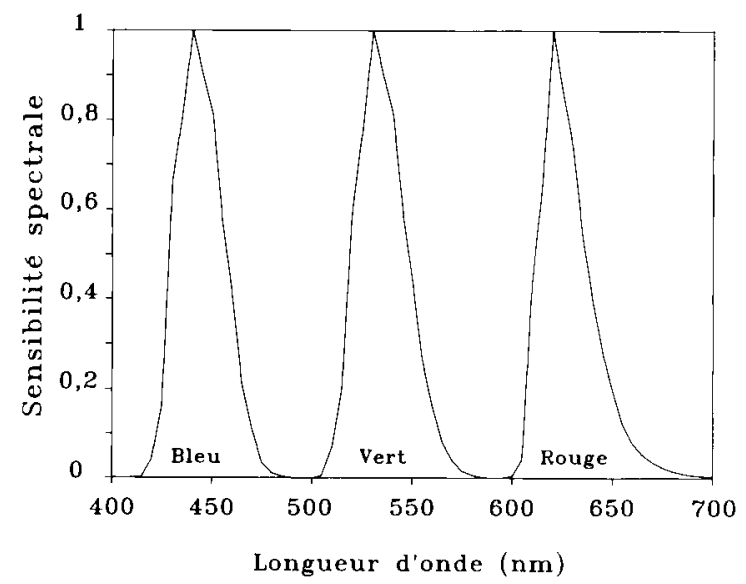

Fig 1. Transmission spectrale des filtres du densitomètre MAC-BETH (Status A). (fig 2) et associées chacune à un pigment (cyan, magenta, jaune). La mesure, par un densitomètre scanneur, de la densité optique du cliché dans les bandes d'absorption de chaque pigment permet d'utiliser le film comme un détecteur. On trouvera dans Scarpace (1978) une description des concepts de base pour l'étalonnage de ce type de capteur. La procédure retenue nécessite d'établir 3 relations :

- la relation d'étalonnage du densitomètre scanneur;

- la relation entre la densité optique et l'exposition lumineuse reçue par le film;

- la relation entre l'exposition lumineuse reçue par le film et la réflectance de la cible.

\section{Étalonnage du densitomètre scanneur}

L'étalonnage est plus complexe pour des films couleurs que pour des films noir et blanc, car les densités mesurées dépendent des caractéristiques précises des filtres du densitomètre par rapport aux bardes spectrales d'absorption des pigments du film. La norme de référence pour les bandes passantes des filtres est la norme Status A (fig 1); toutefois celle-ci n'est généralement pas utilisée pour les densitomètres scanneurs, car la vitesse d'acquisition élevée impose des filtres à bande passante plus large. Pour calculer les densités Status A du film à partir des mesures du densitomètre scanneur nous avons ajusté une relation linéaire :

$$
D^{j}=D_{0}^{j}+\sum_{k=1}^{3} A^{k, j} \times M^{k}
$$

Les indices $j$ et $k$ sont relatifs à la couche du film considérée,

$D i$ : densité optique Status $\mathrm{A}$,

$M^{k}$ : mesure délivrée par le densitomètre scanneur, $A^{k, j}$ et $D_{o}^{j}$ : coefficients à ajuster.

Les coefficients $A^{k, j}$ et $D_{0}^{j}$ sont ajustés expérimentalement selon la méthode proposée par Sant (1970). Sur chaque film, on prend une photographie d'une palette de 14 couleurs; cette photographie est numéri-

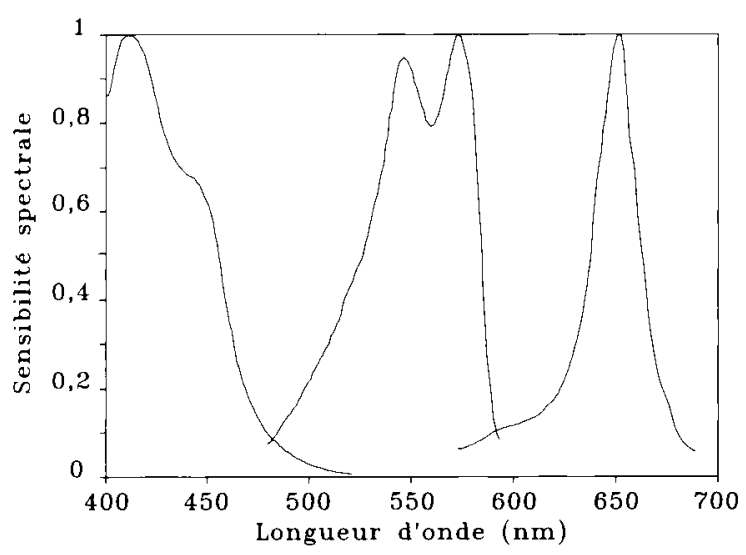

Fig 2. Sensibilité spectrale du film photographique utilisé (Fuji RTP 704) (à partir des feuillets techniques Fuji). 
sée d'une part avec le densitomètre scanneur et d'autre part avec le densitomètre MAC-BETH dont les filtres sont conformes à la norme Status $A$. Les coefficients $A^{k, j}$ sont alors estimés par régression.

\section{Relation entre la densité optique (D) et l'exposition lumineuse (Q)}

La fonction de réponse du film à l'exposition lumineuse, $D=f(Q)$, est fournie dans la documentation du fabricant pour des conditions standard de développement. Pour tenir compte de la variabilité d'un lot de films à l'autre, nous avons mesuré expérimentalement cette fonction. Sur chaque film, on photographie une charte étalon présentant 12 plages de transmittance connues, placée sur une table lumineuse homogène. La table lumineuse n'étant pas étalonnée, la relation expérimentale ainsi établie permet de calculer $Q$ à une constante multiplicative près, à partir des densités optiques du cliché :

$$
Q_{i}^{j}=\mathrm{B} j \times\left(f^{i} j\right)^{-1}\left(D_{i}^{j}\right)
$$

$i$ est relatif au point considéré et $j$ à la couche du film, $f^{\prime}$ : fontion établie empiriquement, donnant la densité optique en fonction de la transmittance de la charte étalon. f' est mesurée expérimentalement pour les 12 plages de la charte de transmittance, et interpolée linéairement pour les valeurs intermédiaires,

$B$ : constante (inconnue) telle que $f^{\prime}=B \times t$.

\section{Relation entre l'exposition du film et la réflec-} tance de la cible

L'exposition lumineuse reçue par le film dépend de la réflectance de la cible, de l'éclairement incident sur celle-ci, et des paramètres de prise de vue (durée d'exposition, rendement lumineux de l'objectif photographique) :

$$
Q_{p i}^{j}=K_{p i}^{j} \times E_{p i}^{j} \times R_{i}^{j}
$$

i est relatif au point considéré et $j$ à la couche du film; $p$ est relatif à la photographie,

$R_{i}^{j}$ : réflectance de la cible,

$E_{p i}^{j}$ : éclairement incident sur la cible,

$K_{p i}^{j}$ : durée d'exposition $\mathrm{x}$ rendement lumineux de l'objectif.

Les termes $K$ et $S$ sont indicés par $i$ et $p$ car ils sont susceptibles de varier d'un point à l'autre d'un même cliché (perte de luminosité entre le centre et les bords du cliché, distance de la feuille à la source lumineuse), et d'un cliché à l'autre (variations dans l'alimentation du dispositif d'éclairage, ou la vitesse d'obturation de l'appareil photographique). On peut distinguer ces 2 sources de variations en considérant $K_{p i}^{j}$ et $E_{p i}^{j}$ comme le produit de leur valeur au centre du cliché (notée $K_{p o}^{j}$ et $E_{p o}^{\prime}$ ) et d'une fonction traduisant les variations spatiales (notée $K_{i}^{j}$ et $E_{j}^{\prime}$ ). En faisant cette décomposition, et en combinant (2) et (3) on obtient :

$$
\left(f^{\prime} j\right)^{-1}\left(D_{i}^{j}\right)=K_{p o}^{j} \times E_{p o}^{j} \times(1 / \mathrm{B} /) \times K_{i}^{j} \times E_{i}^{j} \times R_{i}^{j}
$$

- $f^{\prime j} j$ est la fonction de la relation (2),

- $K_{i}^{j} \times E_{j}^{j}$ est calculé en photographiant une surface lambertienne (ie de réflectance isotrope) placée sur le support; pour une telle surface, $R_{i}^{i}$ ne dépend pas du point considéré. En appliquant la relation (4) au centre et en un point $i$ du cliché on obtient donc : $K_{i}^{d} \times E_{i}^{j}=$ $\left.\left[\left(f^{\prime} j\right)^{-1}\left(D_{i}^{j}\right)\right] /\left[\left(f^{\prime j}\right)^{-1} D_{o}^{j}\right)\right]$.

$-D_{o}^{j} \times E_{p o}^{j} \times(1 / \mathrm{Bj})$ est calculé pour chaque cliché, en plaçant au centre du support une petite surface de réflectance connue : en appliquant la relation (4) aux densités mesurées sur l'image de cette surface on obtient :

$$
\left.K_{p o}^{j} \times E_{p o}^{j} \times(1 / \mathrm{B})=\left(1 / R_{i}^{j}\right) \times\left(f^{\prime}\right)\right)^{-1}\left(D_{i}^{j}\right)
$$

\section{Résumé du protocole expérimental d'étalonnage}

Sur chaque film, on photographie une palette de 12 couleurs pour pouvoir calculer les coefficients $A^{k, j}$, puis la charte étalon de transmittance pour le calcul de $f '$, enfin une surface lambertienne couvrant tout le champ de l'objectif, pour le calcul de $K_{i}^{j} \times E_{i}^{j}$. On réalise ensuite les photographies de feuilles; une petite surface étalon apparait au centre de chaque photo pour permettre le calcul de $K_{p o}^{j} \times E_{p o}^{j} \times(1 / \mathrm{B})$.

\section{RÉSULTATS}

\section{Relations entre les concentrations des différents pigments}

Le dosage direct des chlorophylles $\left(C_{\mathrm{a}}, C_{\mathrm{b}}\right)$ et des caroténoïdes $\left(X_{c}\right)$ montre des relations linéaires entre les concentrations des pigments, qui sont indépendantes de l'origine de la chlorose (jaunisse ou herbicide) :

$$
\begin{array}{ll}
C_{\mathrm{b}}=0,29 \times C_{\mathrm{a}} & \left(n=33, r^{2}=0,95\right) \\
X_{\mathrm{c}}=1,6+0,18 \times\left(C_{\mathrm{a}}+C_{\mathrm{b}}\right) & \left(n=33, r^{2}=0,94\right)
\end{array}
$$

Ces relations sont similaires à celles obtenues pour du blé (Andrieu et Baret, 1988) : $C_{b}=0,35$ $\times C_{\mathrm{a}} ; X_{\mathrm{c}}=3+0,20 \times\left(C_{\mathrm{a}}+C_{\mathrm{b}}\right)$.

Compte tenu que les domaines d'absorption des chlorophylles $a$ et $b$ se recouvrent fortement (Bruinsma, 1981) et que $C_{\mathrm{a}}$ et $C_{\mathrm{b}}$ sont très liées, on se réfèrera dans la suite à la concentration totale en chlorophylles, notée $C_{\mathrm{ab}}=C_{\mathrm{a}}+C_{\mathrm{b}}$. D'autre part, bien que l'absorption par les caroténoïdes dans le vert ne soit pas négligeable devant celle due aux chlorophylles (fig 3 ), la relation entre $C_{\mathrm{ab}}$ et $X_{\mathrm{c}}$ permet d'utiliser le vert pour l'estimation des chlorophylles. Nous avons envi- 


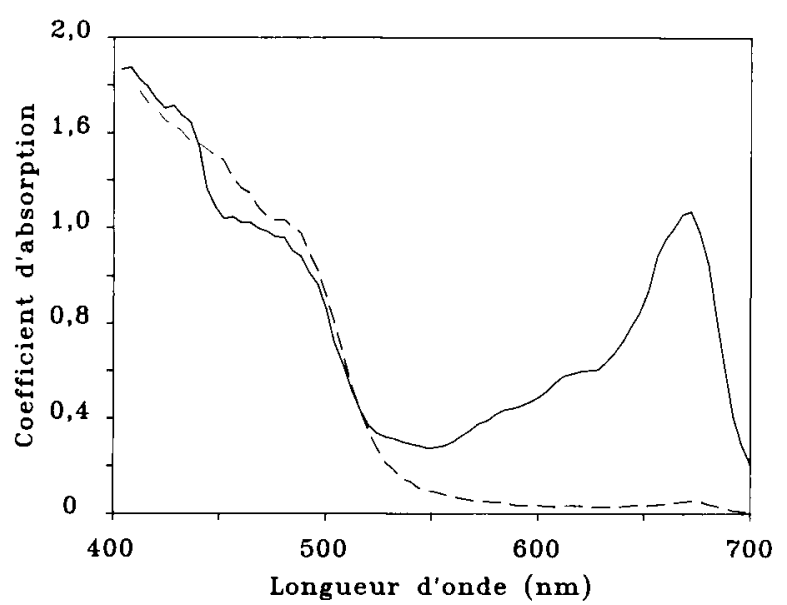

Fig 3. Spectres d'absorption des pigments foliaires - chlorophylles $\mathrm{a}+\mathrm{b}\left(\mathrm{C}_{\mathrm{ab}}: 40 \mu \mathrm{g} / \mathrm{cm}^{2}\right) ;---$ Caroténoïdes totaux $\left(X_{c}: 9 \mu \mathrm{g} / \mathrm{cm}^{2}\right)$; (d'après les données utilisées dans PROSPECT).

sagé dans la suite l'utilisation du vert et du rouge, en effet :

- l'absorption du rayonnement par les chlorophylles est plus importante dans le rouge que dans le vert : les mesures réalisées dans le rouge auront donc une meilleure sensibilité pour les faibles concentrations en pigment. L'utilisation du rouge permet en outre de s'affranchir de la relation entre $C_{\mathrm{ab}}$ et $X_{\mathrm{c}}$, car, dans cette bande spectrale, le coefficient d'absorption des caroténoïdes est très faible devant celui des chlorophylles;

- dans le cas de fortes concentrations en chlorophylles, la valeur élevée du coefficient d'absorption dans le rouge conduit à une saturation du signal. L'utilisation du vert peut alors être préférable.

\section{Simulations sur modèle et mesures directes de la réflectance foliaire}

On a simulé les propriétés optiques des feuilles dans le rouge $(650 \mathrm{~nm})$ et le vert $(550 \mathrm{~nm})$ en fonction de la concentration en pigments et du paramètre de structure $(N)$. Le paramètre $N$ peut être estimé en mesurant la réflectance et la transmittance dans le proche infrarouge; $N=3$ correspond à une feuille très épaisse $(R=0,60$ et $T=0,30$ dans le proche infrarouge (plR)), $N=1$ correspond à une feuille très fine $(R=0,32$ et $T=$ 0,66 dans le plR). Pour les feuilles de betteraves que nous avons analysées, la gamme de variation de $N$ est de 1,4 à 1,8. Dans nos simulations, la concentration en caroténoïdes dépend de la concentration en chlorophylles selon la relation empirique établie plus haut, toutefois on a vérifié que le choix d'une valeur fixe pour $X_{\mathrm{c}}\left(10 \mu \mathrm{g} / \mathrm{cm}^{2}\right)$ ne modifiait pas significativement les résultats des simulations dans le rouge (données non présentées).

La figure 4 montre les relations entre $1 / R_{650}$ et $C_{\mathrm{ab}}$, ainsi qu'entre $1 / R_{550}$ et $C_{\mathrm{ab}}$, pour des valeurs de $C_{\mathrm{ab}}$ comprises entre 0 et $70 \mu \mathrm{g} / \mathrm{cm}^{2}$. Cette relation est très peu sensible au paramètre de structure pour les valeurs habituelles de celui-ci, la sensiblité à $N$ ne devenant importante que pour $N<1$, ce qui peut correspondre au cas de feuilles juvéniles : la sensibilité est alors plus grande à $550 \mathrm{~nm}$ qu'à $650 \mathrm{~nm}$.

Pour comparer les mesures aux prédictions du modèle, les simulations ont été faites pour les bandes spectrales du densitomètre (centrées sur $625 \mathrm{~nm}$ et $535 \mathrm{~nm}$ ), avec une valeur $N=1,6 \mathrm{du}$ paramètre de structure. Le coefficient d'extinction des chlorophylles étant plus faible à $625 \mathrm{~nm}$ qu'à $650 \mathrm{~nm}$, la relation entre $1 / R$ et $C_{\mathrm{ab}}$ devient pratiquement linéaire dans le rouge et le vert, pour la gamme des concentrations en chlorophylle mesurées sur nos feuilles $\left(0-60 \mu \mathrm{g} / \mathrm{cm}^{2}\right)$. La figure 5 compare ces simulations avec la réflectance foliaire mesurée pour différentes valeurs de $C_{\mathrm{ab}}$. Les résultats expérimentaux confirment l'existence d'une relation linéaire entre $1 / R$ et $C_{\mathrm{ab}}$, et la très faible dispersion montre que cette relation est utilisable pour l'estimation de la concentration en chlorophylle à partir d'une mesure de réflectance. Les droites d'ajustement des points expérimentaux sont données ci-dessous :

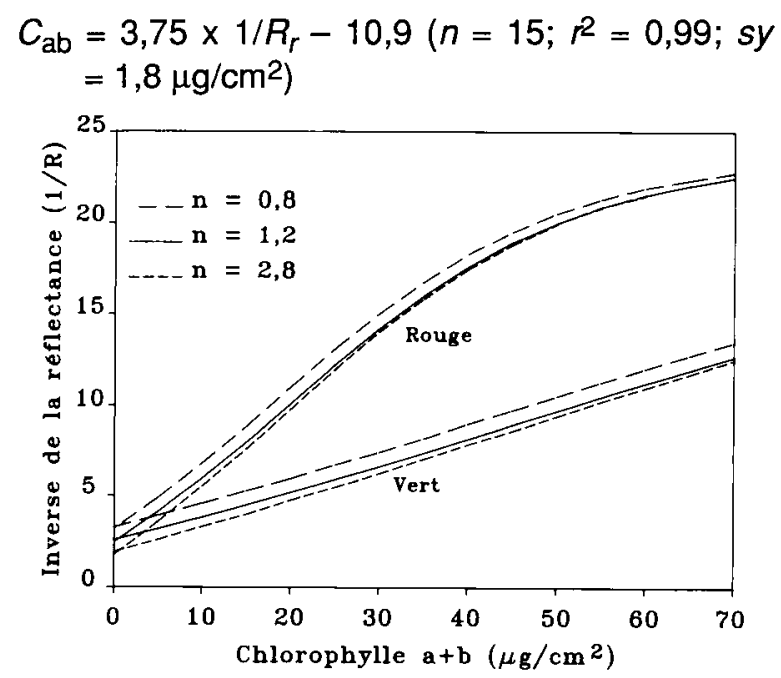

Fig 4. Évolution de l'inverse de la réflectance foliaire à 650 $\mathrm{nm}$ et à $550 \mathrm{~nm}$, en fonction de la concentration en chlorophylles, pour 3 valeurs du paramètre de structure $(N=0,8$ 1,2 et 2,8); (simulations avec le modèle PROSPECT). 


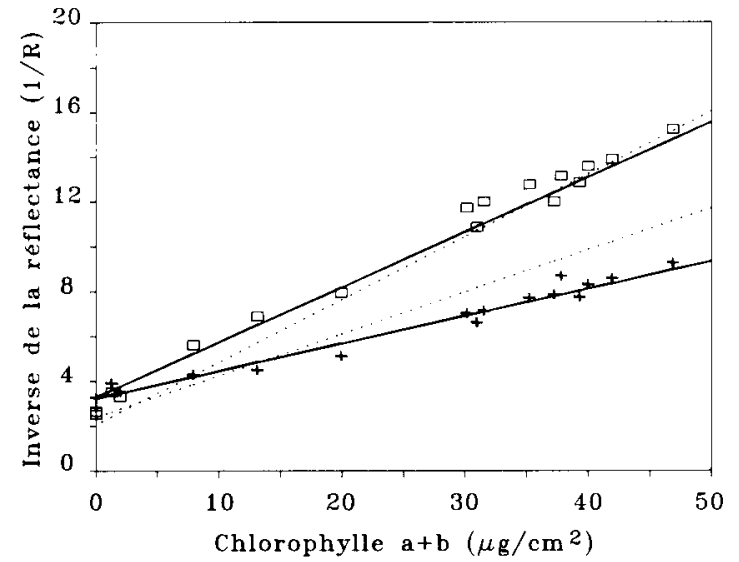

Fig 5. Relation entre l'inverse de la réflectance foliaire et la concentration en chlorophylles, pour les bandes spectrales du densitomètre. $\square$ rouge (mesures); + vert (mesures); ajustements des points expérimentaux; - - - simulations.

$$
\begin{aligned}
C_{\mathrm{ab}} & =7,67 \times 1 / R_{v}-23,5\left(n=15 ; r^{2}=0,97 ; s y\right. \\
& \left.=2,8 \mu \mathrm{g} / \mathrm{cm}^{2}\right)
\end{aligned}
$$

Pour le rouge, les ajustements expérimentaux sont assez proches de ceux obtenus par simulation; par contre dans le vert on observe un écart important entre la droite ajustée expérimentalement et les simulations. Ces différences proviennent en partie des limitations du modèle : d'une part celui-ci tend à sous-estimer les faibles valeurs de réflectance (Jacquemoud et Baret, 1991), d'autre part la réflectance est mesurée ici de façon directionnelle (rayons incidents et réfléchis proches de la normale à la feuille), alors que le modèle prédit la réflectance hémisphérique : des écarts peuvent donc survenir du fait que les feuilles ne sont pas des réflecteurs lambertiens. Un autre élément d'explication est la sensibilité des paramètres d'ajustement vis-à-vis

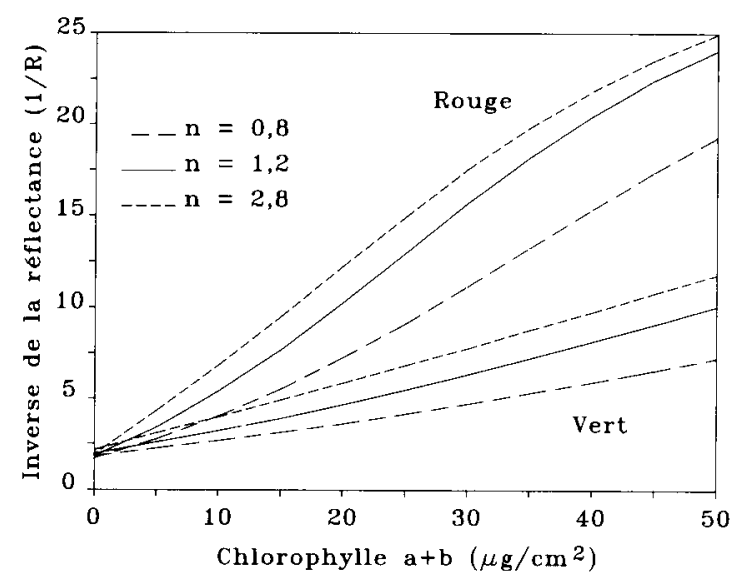

du coefficient d'extinction des chlorophylles, donc de la bande spectrale considérée. Nos calculs ont été faits pour les bandes spectrales nominales de la norme Status $A$, et non sur la base de mesures expérimentales de la transmittance des filtres utilisés.

\section{Utilisation de la photographie numérisée}

\section{Étalonnage}

Les coefficients $A^{k, j}$ de la relation (1) ont été ajustés expérimentalement pour une gamme de densités optiques allant de 0 à 2 :

$D^{r}=0,16+0,935 M^{r}+0,245 M^{v}-0,060 M^{b}(n=$ $14 ; r^{2}=0,997$ )

$D^{v}=0,14-0,111 M^{r}+1,156 M^{v}-0,068 M^{b}(n=$ $14 ; r^{2}=0,996$ )

\section{$r$ : rouge; $v$ : vert; $b$ : bleu.}

Les fonctions de réponse du film à l'exposition lumineuse obtenues expérimentalement sont conformes aux courbes nominales données par le fabricant (données non présentées).

\section{Estimation de la concentration en chlorophylle}

Dans le cas des photographies de feuilles, le signal mesuré ne provient pas uniquement de la réflectance foliaire, il existe une contribution du signal transmis par la feuille et réfléchi par le support blanc. La réflectance apparente est donc (au $2^{e}$ ordre) $R_{f}^{*}=R_{f}+R_{s} \times T_{f}^{2}$, où $R_{f}$ et $T_{f}$ sont la réflectance et la transmittance foliaires, et $R_{S}$ la réflectance du support. La figure 8 montre que

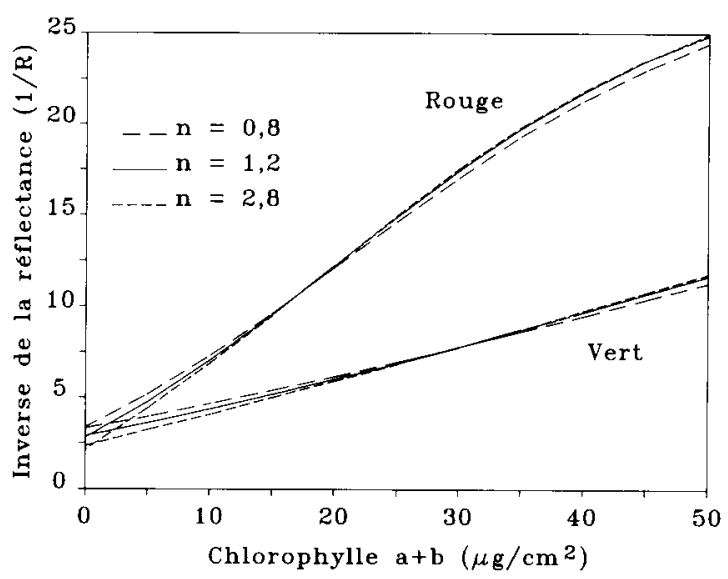

Figs 6,7 . Évolution de la réflectance apparente estimée par photographie en fonction de la concentration en chlorophylles, pour 3 valeurs du paramètre de structure $(N=0,81,2$ et 2,8$)$, et 2 supports (fig $6: R s=1$; fig $7: R s=0,15$ ). 


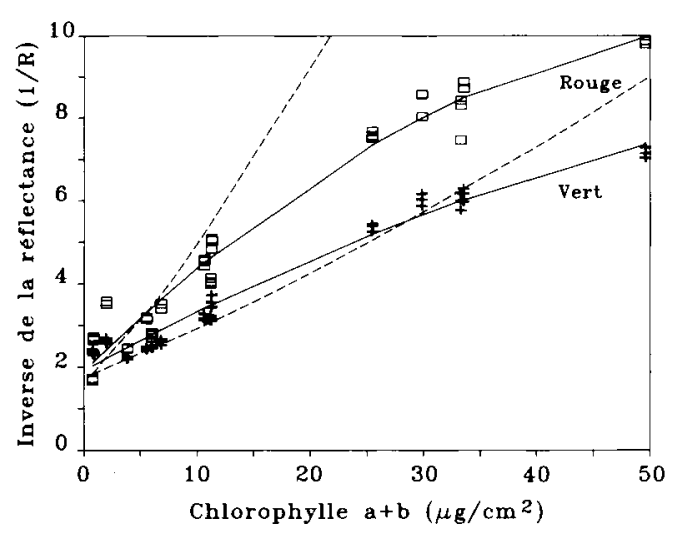

Fig 8. Relation entre la réflectance apparente mesurée par photographie des feuilles (support blanc) et la concentration en chlorophylles. $\square$ rouge; + vert; - ajustements des points expérimentaux; - - - simulations pour $650 \mathrm{~nm}$ (rouge) et $550 \mathrm{~nm}$ (vert).

pour $R_{s}=1$, la sensibilité à la structure est accentuée dans le vert pour les faibles valeurs de $N$. Compte tenu de la gamme effective de variation du paramètre de structure $(1,4$ et 1,8$)$, son incidence sur l'estimation de $C_{\mathrm{ab}}$ reste très faible dans le rouge; en outre la figure 9 , correspondant à $R_{s}=0,15$ montre que cet effet peut être presque totalement éliminé en adaptant la réflectance du support.

Dans notre cas, un fond blanc $\left(R_{S}=1\right)$ a été utilisé; la figure 8 présente la relation entre la concentration en chlorophylles et la réflectance dans le rouge et le vert, estimée à partir des densités optiques du film. On observe que la pente des relations est plus faible dans le vert et sur-

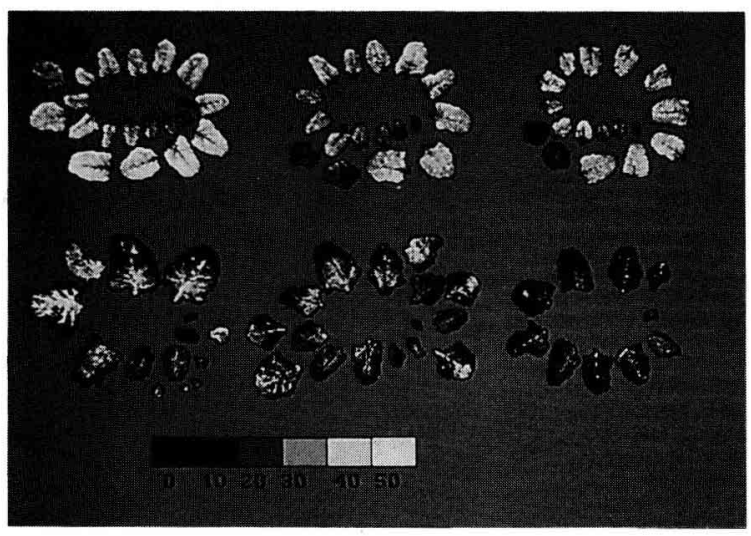

Fig 9. Distribution des concentrations foliaires en chlorophylles, estimées par traitement numérique, pour 6 photographies de plantes. Les valeurs sur l'échelle de gris correspondent aux concentrations surfaciques exprimées en $\mu \mathrm{g} / \mathrm{cm}^{2}$. tout dans le rouge que celle résultant des mesures directes ou des prévisions du modèle; d'autre part il apparaît une déviation par rapport à la linéarité observée précédemment : les points expérimentaux peuvent être ajustés par un polynome du second degré avec une faible dispersion :

$C_{\mathrm{ab}}=0,15+0,47 \times(1 / R r)^{2}\left(n=45 ; r^{2}=0,968 ;\right.$ sy $\left.=2,7 \mu \mathrm{g} / \mathrm{cm}^{2}\right)$

$C_{\mathrm{ab}}=-1,4-0,95 \times(1 / R v)^{2}\left(n=45 ; r^{2}=0,974\right.$; $\left.s y=2,4 \mu \mathrm{g} / \mathrm{cm}^{2}\right)$

Les imprécisions de mesure au cours du processus d'étalonnage du film peuvent expliquer les différences des courbes d'ajustement par rapport au cas de mesures directes. L'élément le plus important est probablement la sélectivité spectrale : d'une part la bande spectrale de sensibilité des pigments du film est plus large que la bande passante des filtres utilisés pour les mesures directes (figs1 et 2), d'autre part, lors de la numérisation, la mesure de la densité d'une couche est affectée par la densité des autres couches du film (Sant, 1970) : ainsi il existe une contribution non négligeable de la couche verte (et même de la couche bleue) à la mesure réalisée dans le rouge. Ceci peut expliquer que la baisse de sensibilité soit plus importante dans le rouge que dans le vert.

La dispersion autour des courbes d'ajustement a plusieurs origines: les imprécisions dans l'interétalonnage des clichés, la plus grande sensibilité à l'indice de structure; toutefois l'élément le plus important est sans doute la non planéité des feuilles : l'éclairement de la cible, qui intervient dans la relation (3), dépend de l'angle entre le rayon incident et la normale à la feuille. La relation (3) est utilisée pour calculer la réflectance foliaire à partir de l'exposition lumineuse du film. Les défauts de planéité des feuilles conduisent donc à une imprécison dans l'estimation de la réflectance.

Malgré les limitations précédentes, la dispersion autour de la courbe d'ajustement reste faible. La relation ajustée sur l'échantillon d'apprentissage a été appliquée à l'ensemble des clichés, soit 60 plantes ( 3 traitements $\times 4$ répétitions $\times 5$ plantes) : la figure 9 présente un exemple de résultats de l'analyse. Le tableau I montre que les concentrations en pigment estimées sur plusieurs répétitions d'un même traitement diffèrent de moins de $3 \mu \mathrm{g} / \mathrm{cm}^{2}$ par rapport à la moyenne du traitement. 
Tableau I. Concentrations moyennes en chlorophylle estimées à partir de photographies: estimations minimale et maximale réalisées sur 4 répétitions de chaque traitement. ( $\mathrm{HO}$ : témoin; $\mathrm{H} 1: 1,6 \mathrm{~g} / \mathrm{ha}$ de Metsulfuron méthyle; $\mathrm{H2}: 2,4 \mathrm{~g} / \mathrm{ha}$ de Metsulfuron méthyle).

\begin{tabular}{ll}
\hline Traitement & $C_{a b}\left(\mu \mathrm{g} / \mathrm{cm}^{2}\right)$ \\
\hline $\mathrm{H} 2$ & $11,7-14,5$ \\
$\mathrm{H} 1$ & $20,5-25,1$ \\
$\mathrm{H} 0$ & $29,7-35,1$ \\
\hline
\end{tabular}

\section{DISCUSSION ET CONCLUSION}

Les mesures réalisées confirment la forme prédite par le modèle pour la relation entre réflectance et concentration en chlorophylles; toutefois les paramètres ajustés diffèrent sensiblement de ceux obtenus par simulation. Cette différence semble provenir principalement du fait que les capteurs que nous avons utilisés privilégient la rapidité d'acquisition par rapport à un contrôle parfait de la géométrie de la mesure et de la sensibilité spectrale.

Malgré les limitations évoquées, la faible dispersion des points expérimentaux autour des courbes d'ajustement montre qu'il est possible d'estimer la concentration en chlorophylles à partir d'une seule mesure de réflectance dans le rouge ou le vert, à condition de disposer d'un échantillon d'apprentissage pour l'ajustement de 2 paramètres. Dans le cas des mesures directes de réflectance avec le densitomètre MAC-BETH, la gamme de sensibilité est de l'ordre de $0-50 \mu \mathrm{g} / \mathrm{cm}^{2}$ pour le rouge et au moins de $0-60 \mu \mathrm{g} / \mathrm{cm}^{2}$ pour le vert, ce qui doit convenir à la majorité des applications.

Des appareils portables existent pour l'estimation indirecte de la concentration en chlorophylles : Le Chlorophyll Meter (Mozaic System) utilise une mesure de transmittance dans le rouge, le SPAD 502 (Minolta) utilise le rapport des transmittances dans le rouge et le proche infrarouge. Dans les 2 cas, l'estimation est réalisée à partir d'une relation empirique entre la transmittance et la concentration en chlorophylles. La précision des estimations est toutefois limitée par l'influence de la structure de la feuille sur la transmission (Hardacre et al, 1984; Yadava, 1986); d'autre part, les mesures ne peuvent pas être réalisées sur des feuilles de forte épaisseur. Les estimations à partir de mesures de réflectance foliaire présentent l'intérêt d'être peu sensibles à la structure de la feuille. L'appareil MAC-BETH utilisé est conçu pour une utilisation en laboratoire; les résultats obtenus montrent qu'il serait intéressant de développer un appareil portable utilisant des mesures de réflectance.

L'intérêt de la procédure proposée est plus particulièrement de pouvoir acquérir les mesures sous forme d'images pour obtenir une description spatiale de la distribution des pigments. Lors de l'utilisation de photographies numérisées, la gamme de sensibilité est environ $0<C_{a b}<50$ $\mu \mathrm{g} / \mathrm{cm}^{2}$, d'autre part, la non planéité des feuilles augmente un peu la dispersion des mesures; toutefois l'erreur quadratique moyenne est suffisamment faible pour permettre d'utiliser la photographie numérisée lorsque l'on souhaite une analyse détaillée de grands échantillons $\left(30 \mathrm{~m}^{2}\right.$ de tissus foliaires ont été analysés dans notre cas). L'acquisition des images sous forme directement numérique avec une caméra CCD permettrait sans doute d'obtenir des résultats équivalents, tout en améliorant la sélectivité spectrale et en simplifiant le processus d'étalonnage.

\section{REMERCIEMENTS}

Cette étude a été réalisée dans le cadre d'une collaboration entre I'INRA, I'AFRC et l'université de Nottingham, avec le soutien financier de PNTS et du NERC. Nous remercions M Steven, T Malthus et $J$ Eastwood pour les mesures de concentrations en pigments, $S$ Jacquemoud et $\mathrm{F}$ Baret pour la mise à disposition du modèle PROSPECT.

\section{RÉFÉRENCES}

Allen WA, Gausman HW, Richardson AJ, Thomas JR (1969) Interaction of isotropic light with a compact plant leaf. J Opt Soc Am 60, 542-547

Andrieu B, Baret F (1988) Estimation de spectres de feuilles à partir de mesures en bandes spectrales larges. In: 4e Coll int Signatures spectrales d'objets en télédétection. (TD Guyenne, JJ Hunt, eds), Aussois (France), 18-22 Janvier 1988, ESA SP-287, Paris, 351-356

Bruinsma J (1961) A comment on the spectrophotometric determination of chlorophyll. Biochim Biophys Acta 52, 576-578

Eastwood JA, Steven MD (1991) The effect of the distribution of chlorotic material on the reflectance of mixed canopies. In: $5^{e}$ Coll Int Mesures physiques 
et signatures en télédétection, Courchevel (France), 14-18 janvier 1991, ESA SP-319, Paris, 397-400

Hardacre AK, Nicholson HF, Boyce MLP (1984) A portable photometer for the measurement of chlorophyll in intact leaves. $N Z J$ Exp Agric 12, 357-362

Jacquemoud S, Baret F (1991) PROSPECT: A model of leaf optical properties spectra. Remote Sensing Environ 34, 75-91

Lichtenthaler HK, Wellburn AR (1983) Determination of total carotenoids and chlorophylls $a$ and $b$ of leaf extracts in different solvents. Biochem Soc Trans $603,591-592$

Sant AJ (1970) Procedures for equivalent neutral density; calibration of color densitometers using a digital computer. Photograph Sci Eng 14, 356-362

Scarpace FL (1978) Densitometry on multi-emulsion imagery. Photogram Eng Remote Sens 44, 1279-1292

Yadava UL (1986) A rapid and non destructive method to determine chlorophyll in intact leaves. Hortsci 21, 1449-1450 